mehrjährigen Erfahrungen wage ich es auszusprechen, dass eine dermaassen stabile Aufstellung sich für Refractoren sehr wohl erreichen lässt, dass Zonenbeobachtungen mit einem Registrirmikrometer gute Sternpositionen liefern. Ganz be-

Berlin, 1898 Febr. 25. sonders wird fur eine sichere Lagerung der Stundenaxe in der beschriebenen Weise und für zuverlässige Klemmvorrichtungen an beiden Axen gesorgt werden müssen.

\title{
Die rotatorische Bewegung des rothen Jupiterflecks.
}

\author{
Von $O$. Lohse.
}

[Mit einer Tafel.]

Der nun seit ungefähr 20 Jahren in $20^{\circ}$ südlicher jovigraphischer Breite sichtbare ovale Fleck war der Gegenstand zahlreicher Beobachtungen, die sich in den meisten Fällen auf die Durchgänge des Flecks durch die Mitte der Scheibe bezogen. Das Material zu einer genatuen Untersuchung der Umdrehungszeit dieses Gebildes war daher vorhanden und die Durchsicht desselben ergab, dass die Rotationszeit nicht constant war. Die Ephemeriden wurden mehrfach um namhafte Beträge geändert, um sie zukünftigen Untersuchungen anzupassen.

Die Bearbeitung meiner eigenen Beobachtungen zielte darauf $a b$, von der Variation der Umdrehungszeit, resp. von der indicirten eigenen Bewegung des Flecks in der Atmosphäre des Jupiter ein möglichst klares und deutliches Bild zu erhalten. Ich wich daher von der üblichen Methode der Bestimmung von Umdrehungszeiten für verschiedene Epochen $\mathrm{ab}$, und berechnete aus zahlreichen Beobachtungen für jede Opposition einen Normalort des Mittelpunktes des Flecks auf dem Jupitersphäroid, unter der Annahmre eines festen Meridians und einer gleichförmigen Rotationsgeschwindigkeit des Planeten.

Die erwähnten Aenderungen der Ephemeriden von A. Marth, durch constante Beträge sowohl als durch Zugrundelegung verschiedener täglicher Rotationswinkel waren bei der Rechnung sehr störend und mussten erst wieder compensirt werden.

Obgleich diese Untersuchung einen Theil einer Arbeit uber Jupiter bildet, die noch nicht abgeschlossen ist, so möchte ich doch schon jetzt über das Resultat, seiner Merk. würdigkeit wegen, kurz berichten.

Die Berechnung der Normalörter von 1878 ab ergab eine andatuernde Abnahme der jovigraphischen Längen von bemerkenswerther Gesetzmässigkeit, so dass es möglich war, fuir jeden Termin innerhalb der Beobachtungszeiten den Ort des rothen Flecks, resp. seinen Durchgang durch die Mitte der Scheibe, mit ziemlicher Sicherheit $2 u$ berechnen.

Die Eintragungen dieser Normalörter in ein Coordinatensystem, bei dem die Zeiten als Abscissen, die jovigraphischen Längen als Ordinaten angenommen wurden, liessen sich durch eine stetig gekrümmte Curve verbinden, wie aus der beiliegenden Tafel-ersichtlich ist.

Dieses Resultat lag mir bereits 1892 vor, und es war von grossem Interesse, in den folgenden Oppositionen zu beobachten, welchen ferneren Verlauf die Curve nehmen würde.
Da die Oerter immer weniger Verschiedenheit gezeigt hatten, so schien es, als ob allmählig eine gleichförmige Bewegung des Flecks eintreten würde, die vielleicht mit seinem gänzlichen Erlöschen zusammenfiel, denn der Fleck hatte im Laufe der Jahre beträchtlich an Intensität verloren, und wirde zeitweilig schwierig zu verfolgen gewesen sein, wenn nicht eine deutliche conforme Einbuchtung des Aequatorealstreifens seinen Ort gekennzeichnet hätte.

Die ferneren Beobachtungen ergaben nun, dass die jovigraphischen Längen nach $189 \mathrm{x}$ wieder zunehmen, und zwar immer schneller, so dass die letzten vorliegenden Beobachtungen von 1896 und 1897 bereits wieder einen Unterschied von $+10^{\circ}$ zeigen.

Die von mir' für die Jahre 1878 bis 1897 unter $\mathrm{Zu}$ grundelegung des täglichen Rotationswinkels von $870^{\circ} \mathbf{2}$ berechneten jovigraphischen Normallängen des Flecks sind die folgenden:

$\begin{array}{ccc}\text { Epoche } & \begin{array}{c}\text { Jovigr. } \\ \text { Normallänge }\end{array} & \text { Beobachter*) } \\ \text { I878.65 } & 249.5 & \mathrm{~L} \\ 1878.86 & 237.1 & \mathrm{Tr} \\ 1879.73 & 182.7 & \mathrm{~L} \\ 1880.71 & 128.5 & \mathrm{~L} \\ 1881.70 & 89.2 & \mathrm{~L} \\ 1882.14 & 78.0 & \mathrm{~L} \\ 1883.14 & 50.4 & \mathrm{~L} \\ 1884.15 & 32.6 & \mathrm{~L} \\ 1885.27 & 15.8 & \mathrm{~L} \\ 1886.27 & 8.3 & \mathrm{~L} \\ 1887.27 & 2.9 & \mathrm{St}, \mathrm{D} \\ 1888.27 & 358.9 & \mathrm{~L} \\ 1890.15 & 353.6 & \mathrm{~T}, \mathrm{P} \\ 1891.74 & 352.0 & \mathrm{~L} \\ 1892.76 & 356.2 & \mathrm{~L} \\ 1894.03 & 358.8 & \mathrm{~L} \\ 1895.18 & 5.2 & \mathrm{~L} \\ 1896.13 & 10.1 & \mathrm{~L} \\ 1897.27 & 20.4 & \mathrm{~L}\end{array}$

Die beigegebene Tafel lässt auf den ersten Blick die Regelmässigkeit erkennen, mit der sich die jovigraphischen Längen geändert haben. Die in dem angefangenen, aufsteigenden Theile der Curve bemerkbaren Oerter schliessen sich vielleicht nicht ganz so gut an, als in den symmetrisch gelegenen absteigenden Theile, trotzdem in der letzten Zeit die jovigraphischen Längen des Flecks durch zahlreiche

*) $\mathrm{Tr}=$ Trouvelot, $\mathrm{St}=$ Stanley Williams, $\mathrm{D}=$ Denning, $\mathrm{T}=$ Terby, $\mathrm{P}=$ Pritchett, $\mathrm{L}=$ Lohse. 
mikrometrische Messungen bestimmt wurden, während früher hierzu nur Abschätzungen der Passagen des Flecks durch die Mitte der Scheibe dienten. Es kann hieran die geringe Deutlichkeit Schuld sein, mit der die vorangehende Hälfte des Flecks in den letzten Jahren begrenzt war, da diese ungleiche Begrenzung die Abschätzung des Mittelpunktes bei der Messung erschwerte. Aber es ist auch nicht unmöglich, dass hier keine Beobachtungsfehler vorliegen, sondern während der verschiedenen Oppositionen kleinere Abweichungen von der regelmässigen Bewegung stattfanden.

Anscheinend war der Fleck nach seinem Entstehen einer Kraft unterworfen, die ihn in eine verhältnissmässig schnelle Rotation*) versetzte; diese Kraftäusserung verringerte sich Anfangs sehr stark, später nur allmählig, so dass der Fleck gegen das Jahr I89 I hin eine nahezu gleichförmige Bewegung zeigte, die einer normalen täglichen Rotation von $870: 27$ entsprach. Von diesem Zeitpunkte ab trat eine weitere und stärker werdende Verminderung der Rotationsgeschwindigkeit des Flecks ein.

Entspräche der Rotationswinkel von $870: 27$ der wirklichen Umdrehungszeit des festen Jupiterkörpers, so dass der letztere sich in $9^{\mathrm{h}} 55^{\mathrm{m}} 4^{\mathrm{s}}$ um seine Axe drehte, so würden die berechneten Normalörter andeuten, dass der rothe Fleck in 13 Jahren nahezu $3 / 4$ des gesammten Umfanges des Planeten durchwandert hat, dann aber umgekehrt ist und nach und nach wieder die bereits innegehabten Positionen einnimmt.

Da nach der Tafel der aufsteigende Theil der Curve sich symmetrisch zu dem absteigenden gestaltet, so ist die Rotationszeit, welche der Fleck im Jahre 189 I (Scheitel der Curve) gehabt hat, identisch mit der zu Grunde gelegten Rotationszeit. Die letztere wurde von Marth durch fortgesetzte Anpassung der Ephemeriden an die Bewegungen des Flecks erhalten, und ihre Verwerthung für die vorliegende Untersuchung muss als zufällig sehr günstig angesehen werden. Die Beantwortung der Frage, ob dieser Zeit von rund $9^{\mathrm{h}} 55^{\mathrm{m}} 4 \mathrm{I}^{\mathrm{s}}$ eine weitere Bedeutung beigemessen werden kann, wird indess erst möglich sein, wenn es gelingt Ursachen aufzufinden, welche die vorstehend erörterten Bewegungserscheinungen des rothen Flecks, eines der interessantesten Gebilde der Jupiteratmosphäre, zu erklären vermögen.

Potsdam, Astrophysikalisches Observatorium, I 898 Februar.

*) Die Längen werden in einer der Rotation entgegengesetzten Richtung gezählt.

\title{
Beobachtungen von Stern- und Planeten-Bedeckungen durch den Mond
}

\author{
an der k. k. Sternwarte zu Prag.
}

(Mitgetheilt von Prof. Dr. L. Weinek).

Dem Ansuchen des Herrn Prof. Ch. André in Nr. 347 I der Astr. Nachr. entsprechend, erlaube ich mir, im Nachstehenden die bislang noch nicht veröffentlichten Prager Beobachtungen von Stern- und Planeten-Bedeckungen durch den Mond bekannt zu geben. In der Zusammenstellung bedeutet: E. h. Eintritt am hellen, E. d. Eintritt am dunklen, A. h. Austritt am hellen, A. d. Austritt am dunklen Mondrand. Die Abkürzungen für die verwendeten Instrumente sind:

$$
\begin{aligned}
& \mathrm{St}=\text { Steinheil'scher Refractor, Oeffnung }=162.6 \mathrm{~mm} \\
& \mathrm{R}=\text { Reinfelder'scher Refractor, Oeffnung }=108.6 \mathrm{~mm} \\
& \mathrm{Fr}=\text { Fraunhofer'scher Refractor, Oeffnung }=97.6 \mathrm{~mm} \\
& \mathrm{fr}=\text { kl. Fraunhofer'scher Refractor, Oeffnung }=83.7 \mathrm{~mm} \text {. }
\end{aligned}
$$

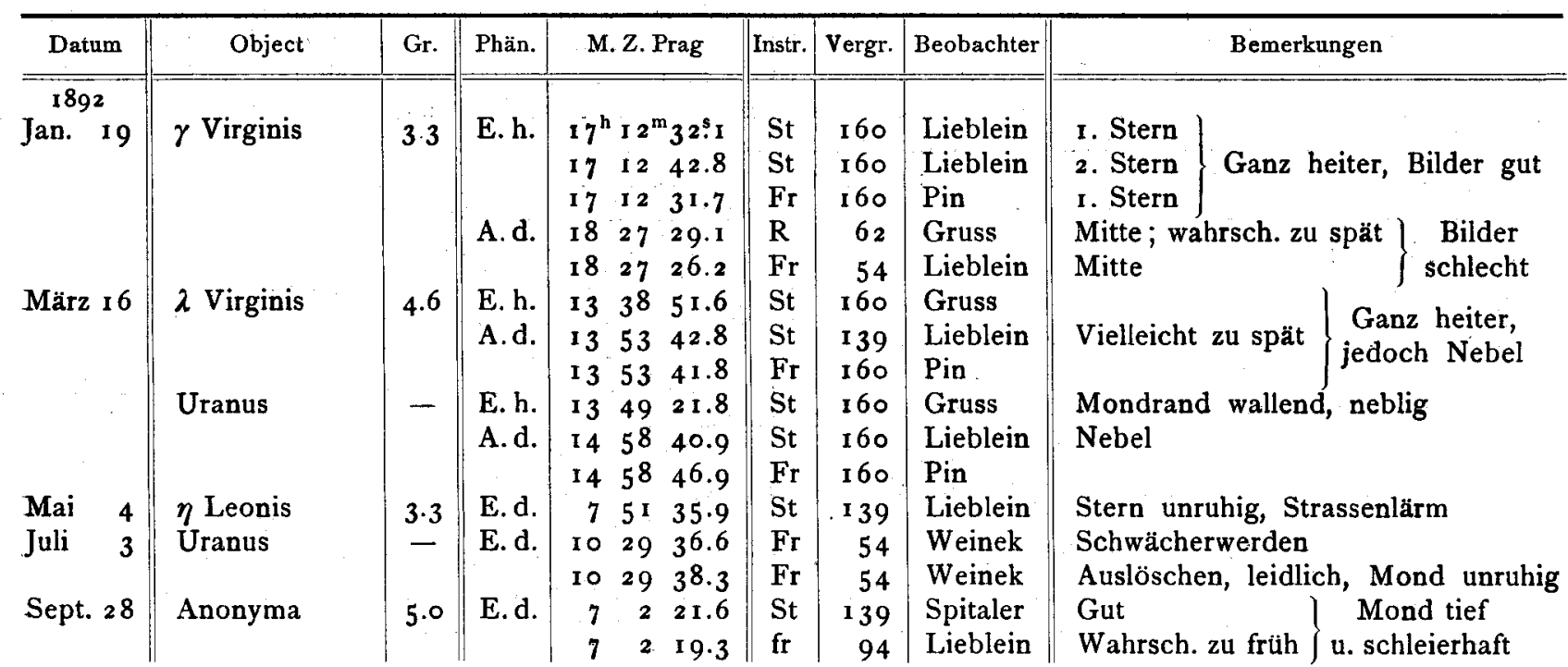

